\title{
A MULTIPLICITY THEORY FOR BOOLEAN ALGEBRAS OF PROJECTIONS IN BANACH SPACES
}

\author{
BY \\ WILLIAM G. BADE $\left({ }^{(1)}\right.$
}

0 . Introduction. Many features of the theory of commutative $W^{*}$-algebras in Hilbert space have been shown to extend to appropriate operator algebras in Banach spaces (cf. $[2 ; 3 ; 7]$ ). Recently an important gap in the theory was closed by Dieudonné [4] who obtained a multiplicity theory in the case that the adjoint $\mathfrak{X}^{*}$ of the underlying Banach space $\mathfrak{X}$ (and hence also $\mathfrak{X}$ ) was separable. In this paper a new approach is developed which does not require assumptions of separability and allows us to construct a multiplicity theory also for the algebra of adjoints in $\mathfrak{X}^{*}$ where the relevant topology is the weak star or $\mathfrak{X}$-topology rather than the norm topology. Of the various treatments of multiplicity theory in Hilbert space our development is perhaps closest to that of Kelley [10].

The analogue of a commutative $W^{*}$-algebra in a Banach space $\mathfrak{X}$ is the operator algebra $\mathfrak{A}$ generated in the uniform topology by a complete Boolean algebra $\mathfrak{B}$ of projections in $\mathfrak{X}$. For given $x \in \mathfrak{X}$, the cyclic subspace determined by $x$ is the manifold $\mathfrak{M}(x)=\operatorname{clm}\{A x \mid A \in \mathfrak{A}\}$. A multiplicity function for $\mathfrak{A}$ is a function $m$ on $\mathfrak{B}$ to cardinal numbers, the value $m(E)$ being roughly the smallest number of cyclic subspaces required to span the range of $E$. The aim of multiplicity theory in this setting is to decompose the identity $I$ in $\mathfrak{B}$ into the sum of disjoint projections $E$ of uniform multiplicity, i.e. projections such that $m(F)=m(E)$, whenever $0 \neq F \leqq E$. In $\$ 2$ such a decomposition theorem is proved to hold for any function on an abstract B.A. to cardinals which satisfies an appropriate condition of order continuity. Multiplicity functions are constructed respectively for $\mathfrak{B}$ in $\mathfrak{X}$ and $\mathfrak{B}^{*}$ in $\mathfrak{X}^{*}$ in $\S \S 3$ and 6 and the continuity condition is verified. Following a detailed analysis of the range of a projection of finite uniform multiplicity, one of the main results of the paper (Corollary 8.5 ) is obtained: A projection $E$ in $\mathfrak{B}$ has finite multiplicity $n$ if and only if its adjoint $E^{*}$ in $\mathfrak{B}^{*}$ has multiplicity $n$. We have been unable to decide whether this result remains true for projections of infinite multiplicity.

Finally an application is made to the theory of spectral operators of scalar type developed by Dunford $[7 ; 8]$. If the resolution of the identity $E(\cdot)$ of

Presented to the Society, April 20, 1957 under the title Multiplicity of spectral measures, I, and on April 26, 1958 under the titles Multiplicity of spectral measures, II, and Similarity equivalence of spectral operators; received by the editors March 13, 1958.

(1) This research was sponsored in part by the Office of Naval Research under Contract Nonr-222(37) (NR041 157). Reproduction in whole or in part is permitted for any purpose of the United States Government. 
such an operator $T$ contains no projections of infinite uniform multiplicity, then $T$ is similarity equivalent, $T=A^{-1} N A$, to a normal operator $N$ on an appropriate Hilbert space $\mathfrak{S}$ where the operator $A: \mathfrak{X} \rightarrow \mathfrak{S}$ and its inverse are closed and densely defined. Moreover, $A$ establishes a one to one correspondence between the subspaces of $\mathfrak{X}$ invariant under $E(\cdot)$ and those of $\mathfrak{S}$ invariant under the resolution of the identity of $N$. This result may be compared with the theorem that every scalar type spectral operator in Hilbert space is similarity equivalent with a normal operator where the operator $A$ yielding the equivalence is bounded. (This important theorem is a consequence of a result of Dixmier [6] and Mackey [11, p. 146].)

1. Preliminaries. For convenience we shall summarize here some facts from the author's paper [3] where further details will be found. A Boolean algebra (B.A.) of projections in a real or complex Banach space is a family $\mathfrak{B}$ of commuting projections containing 0 and $I$ which is a B.A. under the operations

$$
E \vee F=E+F-E F, \quad E \wedge F=E F .
$$

The corresponding ordering is that $E \leqq F$ if and only if $E F=E$. A given family $\left\{E_{\alpha}\right\}$ of elements of $\mathfrak{B}$ may have a least upper bound $\bigvee E_{\alpha}$ or greatest lower bound $\Lambda E_{\alpha}$ in $\mathfrak{B}$, in which case one has the inclusions

$$
\left(\vee E_{\alpha}\right) \mathfrak{X} \supseteq \operatorname{clm}\left\{E_{\alpha} \mathfrak{X}\right\}, \quad\left(\Lambda E_{\alpha}\right) \mathfrak{X} \subseteq \cap E_{\alpha} \mathfrak{X},
$$

which follow directly from the formula for the ordering. (These inclusions are necessarily equalities whenever the family is finite.) The equations

$$
\begin{array}{llrl}
E \wedge\left(\vee F_{\beta}\right) & =\bigvee\left(E \wedge F_{\beta}\right), & & E \in \mathfrak{B}, \\
E \vee\left(\wedge F_{\beta}\right)=\wedge\left(E \vee F_{\beta}\right), & & E \in \mathfrak{B},
\end{array}
$$

are valid whenever the terms are defined $\left({ }^{2}\right)$. We say $\mathfrak{B}$ is complete (or $\sigma$-complete) as an abstract B.A. if every family (sequence) has a least upper bound and greatest lower bound in $\mathfrak{B}$. A stronger form of completeness is more useful, however. In what follows we shall call $\mathfrak{B}$ complete if for every family $\left\{E_{\alpha}\right\} \subseteq \mathfrak{B}$, the projections $\bigvee E_{\alpha}$ and $\Lambda E_{\alpha}$ exist in $\mathfrak{B}$ and, moreover,

$$
\left(\vee E_{\alpha}\right) \mathfrak{X}=\operatorname{clm}\left\{E_{\alpha} \mathfrak{X}\right\}, \quad\left(\Lambda E_{\alpha}\right) \mathfrak{X}=\cap E_{\alpha} \mathfrak{X} .
$$

It is shown in [3, Lemma 2.3] that if $\mathfrak{B}$ is complete, a monotone increasing (decreasing) net $\left\{E_{\alpha}\right\}$ in $\mathscr{B}$ converges in the strong operator topology to $\bigvee E_{\alpha}\left(\Lambda E_{\alpha}\right)$. Similarly one defines a $\sigma$-complete B.A. of projections and a similar convergence theorem holds for monotone sequences. If $\mathfrak{B}$ is $\sigma$-complete (or even only $\sigma$-complete as an abstract B.A.) there is a uniform bound for the norms of the projections in $\mathfrak{B}[3$, Theorem 2.2] which we shall consistently denote by $M$.

${ }^{2}$ See, for example, G. Birkhoff, Lattice theory, New York, 1948, p. 166. 
If $\mathscr{B}$ is $\sigma$-complete, its closure $\overline{\mathfrak{B}}^{s}$ in the strong operator topology is a complete B.A. of projections with the same bound [3, Theorem 2.7]. A $\sigma$-complete B.A. $\mathfrak{B}$ is complete if and only if the algebra generated by $\mathfrak{B}$ in the uniform operator topology is weakly closed [3, Theorem 4.5]. If $\mathfrak{B}$ is complete its Stone space $\Omega$ is extremely disconnected, and we may regard $\mathfrak{B}$ as a projection valued measure $E(\cdot)$ on the Borel sets of $\Omega$. For corresponding to each Borel set $e$ in such a space there is unique open and closed set $e_{0}$ such that $\left(e-e_{0}\right) \cup\left(e_{0}-e\right)$ is of first category. Our definition of completeness insures that the vector measure $E(\cdot) x$ is countably additive for each $x \in \mathfrak{X}$.

Since Dieudonné's results are stated in somewhat different terms from ours it is desirable to compare the two approaches. Let $C(K)$ be the Banach algebra of all continuous complex functions on a compact Hausdorff space and $T$ be a continuous representation of $C(K)$ into the algebra $\mathcal{R}(\mathfrak{X})$ of bounded operators on a separable Banach space $\mathfrak{X}$. For each $x \in \mathfrak{X}, x^{*} \in \mathfrak{X}^{*}$, there exists a regular measure $\mu\left(\cdot ; x, x^{*}\right)$ on the Borel sets of $K$ such that

$$
x^{*} T(f) x=\int_{K} f(k) \mu\left(d k ; x, x^{*}\right), \quad f \in C(K), x \in \mathfrak{X}, x^{*} \in \mathfrak{X}^{*} .
$$

Dieudonné studies such a representation under the hypothesis that $T$ may be extended to a representation (again denoted by $T$ ) of the algebra $B(K)$ of all bounded Borel functions on $K$ into $\mathfrak{\&}(\mathfrak{X})$ such that

$$
x^{*} T(f) x=\int_{K} f(k) \mu\left(d k ; x, x^{*}\right), \quad f \in B(K), x \in \mathfrak{X}, x^{*} \in \mathfrak{X}^{*} .
$$

The range of $T$ on the characteristic functions in $B(K)$ yields a B.A. $\mathscr{B}$ of projections in $\mathfrak{X}$ which we may regard as a spectral measure $E(\cdot)$ on the Borel sets of $K$. Condition (2) implies that $x^{*} E(\cdot) x=\mu\left(\cdot ; x, x^{*}\right)$ so the measures $x^{*} E(\cdot) x$ are countably additive. This in turn implies $\mathfrak{B}$ is $\sigma$-complete and further (since $\mathfrak{X}$ is separable $\mathfrak{B}$ is countably decomposable) that $\mathfrak{B}$ is complete $[3$, p. 350]. Hence the range of $T$ on $B(K)$ is weakly closed. We have preferred to base our development directly on $\mathfrak{B}$.

2. Multiplicity functions on a complete Boolean algebra. This section contains a basic decomposition theorem for certain cardinal number valued functions on a B.A. This theorem is very closely related to a result of Halmos $[9$, p. 82].

2.1. Definition. Let $\mathfrak{B}$ be a complete abstract B.A. A function $m$ on $\mathfrak{B}$ whose values are cardinals will be called a multiplicity function if

$$
\begin{aligned}
m(0) & =0, \text { and } \\
m\left(\bigvee_{\alpha} E_{\alpha}\right) & =\bigvee_{\alpha} m\left(E_{\alpha}\right), \quad\left\{E_{\alpha}\right\} \subseteq \mathfrak{B} .
\end{aligned}
$$

The number $m(E)$ is called the multiplicity of $E$. We say $E \in B$ has uniform multiplicity $n$ if $m(F)=n$ whenever $0 \neq F \leqq E$.

A subset $\mathfrak{D}$ of $\mathfrak{B}$ is an ideal if $E, F \in \mathfrak{D}$ implies $E \vee F \in \mathfrak{D}$ and $G \leqq E, E \in \mathfrak{D}$, 
implies $G \in \mathfrak{D}$. The ideal $\mathfrak{D}$ is dense if every element of $\mathfrak{B}$ is a union of elements of $\mathfrak{D}$. A $\sigma$-ideal is an ideal closed under countable unions.

2.2. Lemma. Let $\mathfrak{D}$ be a dense ideal in $\mathfrak{B}$ and $m$ be a function on $\mathfrak{D}$ to cardinals such that $m(0)=0$ and $m\left(\mathrm{~V}_{\alpha} E_{\alpha}\right)=\mathrm{V}_{\alpha} m\left(E_{\alpha}\right)$ for each family $\left\{E_{\alpha}\right\} \subseteq \mathfrak{D}$ such that $\mathrm{V}_{\alpha} E_{\alpha} \in \mathfrak{D}$. Then there is a unique multiplicity function on $\mathfrak{B}$ which is an extension of $m$ on $\mathfrak{I}$.

Proof. If $E \in \mathfrak{B}$ define $m(E)=\mathrm{V}_{\alpha} m\left(B_{\alpha}\right)$, where $\left\{B_{\alpha}\right\}$ is any family in $\mathfrak{I}$ such that $E=\mathrm{V}_{B_{\alpha}}$. If also $E=\mathrm{V} C_{\beta}, C_{\beta} \in \mathfrak{D}$, then for each $\alpha, \beta, B_{\alpha}=\mathrm{V}_{\beta} B_{\alpha} C_{\beta}$, and $C_{\beta}=\mathrm{V}_{\alpha} B_{\alpha} C_{\beta}$. Thus

$$
\begin{aligned}
\mathrm{V}_{\alpha} m\left(B_{\alpha}\right) & =\mathrm{V}_{\alpha} m\left(\mathrm{~V}_{\beta} B_{\alpha} C_{\beta}\right)=\mathrm{V}_{\alpha, \beta} m\left(B_{\alpha} C_{\beta}\right) \\
& =\mathrm{V}_{\beta} m\left(\mathrm{~V}_{\alpha} B_{\alpha} C_{\beta}\right)=\mathrm{V}_{\beta} m\left(C_{\beta}\right),
\end{aligned}
$$

showing the extension is well defined. To see the extension is a multiplicity function, let $E_{0} \in \mathfrak{b}, E_{0}=\bigvee_{\gamma} E_{\gamma}, E_{\gamma} \in \mathfrak{B}$. For each $\gamma$ we have $E_{\gamma}=\bigvee_{\delta} G_{\gamma \delta}$, where $G_{\gamma \delta} \in \mathfrak{D}$. Then

$$
\begin{aligned}
m\left(E_{0}\right) & =\bigvee_{\gamma, \delta} m\left(G_{\gamma \delta}\right)=\bigvee_{\gamma} \bigvee_{\delta} m\left(G_{\gamma \delta}\right) \\
& =\bigvee_{\gamma} m\left(E_{\gamma}\right) .
\end{aligned}
$$

That the extension is unique follows easily from the distributivity.

2.3. Theorem. Let $m$ be a multiplicity function on a complete abstract $B . A . \mathfrak{B}$. Then there is a unique family $\left\{E_{n}\right\}$ of disjoint elements in $\mathfrak{B}, n$ running over the cardinals $\leqq m(I)$, such that

(i) $I=\bigvee E_{n}$,

(ii) If $E_{n} \neq 0, E_{n}$ has uniform multiplicity $n$.

Proof. We show first that each nonzero $E \in \mathfrak{B}$ bounds a nonzero $G \in \mathfrak{B}$ of uniform multiplicity. For let $n_{0}=\min \{m(F) \mid 0 \neq F \leqq E\}$. Since the cardinals are well ordered, there exists a projection $G \leqq E$ with $m(G)=n_{0}$. Clearly $G$ has uniform multiplicity $n_{0}$. Now from Zorn's lemma we obtain a maximal family $\mathfrak{F}$ of disjoint elements of $\mathfrak{B}$ each having uniform multiplicity. For each cardinal $n$ let $E_{n}$ be the supremum of those $F \in \mathfrak{F}$ with $m(F)=n$. If $0 \neq G \leqq E_{n}$, then $G$ is the union of elements of $\mathfrak{B}$ of multiplicity $n$. Hence $E_{n}$ has uniform multiplicity $n$. Finally suppose also that $I=\bigvee F_{n}$ where the $F_{n}$ are disjoint and satisfy (ii). Suppose $E_{k} \neq 0$. Since we may write $E_{k}$ $=\mathrm{V} E_{k} F_{n}$, each element $E_{k} F_{n}$ is zero or has uniform multiplicity $k$. Thus $E_{k} F_{n}=0$ if $n \neq k$. Consequently $F_{k} \neq 0$ and $E_{k} \leqq F_{k}$. Correspondingly if $F_{k} \neq 0, E_{k} \neq 0$ and $F_{k} \leqq E_{k}$. Thus for each $n, E_{n}=F_{n}$, showing the uniqueness.

3. The multiplicity function for $\mathfrak{B}$ in $\mathfrak{X}$. Now let $\mathfrak{B}$ be a complete B.A. of projections in the real or complex Banach space $\mathfrak{X}$. We shall obtain a natural multiplicity function for $\mathfrak{B}$. To do this a few auxiliary notions will be needed. For each $x \in \mathfrak{X}$ the projection $\Lambda\{E \mid E x=x\}$ will be called the carrier projection of $x$. We note that if $G$ is the carrier projection of $x$ and $0 \neq F \leqq G$, then 
$F x \neq 0$. The cyclic subspace $\mathfrak{M}(x)$ spanned by a vector $x$ is $\operatorname{clm}\{E x \mid E \in \mathfrak{B}\}$. A projection $E \in \mathscr{B}$ will be said to satisfy the countable chain condition if every family of disjoint projections in $\mathfrak{B}$ bounded by $E$ is at most countable. We shall denote by $\mathbb{E}$ the set of all $E \in \mathfrak{B}$ satisfying this condition. The multiplicity will first be defined on $\mathbb{\complement}$, its unique extension to $\mathfrak{B}$ being a consequence of Lemma 2.2.

3.1. Lemma. The set $\mathbb{S}$ is a dense $\sigma$-ideal in $\mathfrak{B}$. A projection belongs to $\mathfrak{S}$ if and only if it is the carrier projection of a vector in $\mathfrak{X}$.

Proof. We note first the purely algebraic fact that (5) is a $\sigma$-ideal. That it is an ideal is clear. Now suppose $F=\mathrm{V}_{n=1}^{\infty} F_{n}$, where $F_{n} \in \mathbb{E}$, and let $F$ be the union of a family $\left\{E_{\gamma} \mid \gamma \in \Gamma\right\}$ of disjoint elements of $\mathfrak{B}$. Then for each fixed $n$, at most countably many of the products $F_{n} E_{\gamma}, \gamma \in \Gamma$, are different from zero. It follows that $\left\{\gamma \mid E_{\gamma} F_{n} \neq 0\right.$ for some $\left.n\right\}$ is at most countable. But this is the set of $\gamma$ for which $E_{\gamma} \neq 0$, since for every $\gamma, E_{\gamma}=\bigvee_{n=1}^{\infty} E_{\gamma} F_{n}$.

Now let $E$ be the carrier projection of a vector $x$ and suppose $E$ is the union of a family $\left\{E_{\alpha}\right\}, \alpha \in A$, of disjoint elements of $\mathfrak{B}$. By [3, Lemma 2.3] we have $\lim _{\sigma} \sum_{\alpha \in \sigma} E_{\alpha} x=x$, where $\sigma$ runs over the finite subsets of $A$, ordered by inclusion. For each $\epsilon>0$ there exists a $\sigma$ such that $\left|x-\sum_{\sigma} E_{\alpha} x\right|<\epsilon M^{-1}$, so if $\beta \notin \sigma$, then $\left|E_{\beta} x\right|=\left|E_{\beta}\left(x-\sum_{\sigma} E_{\alpha} x\right)\right|<\epsilon$. It follows that at most countably many of the vectors $E_{\alpha} x, \alpha \in A$, are not zero. As $E_{\alpha} \leqq E$, the equation $E_{\alpha} x=0$ implies $E_{\alpha}=0$ as remarked above. Thus the carrier of any vector belongs to $\mathfrak{C}$.

It will next be shown that $\mathfrak{C}$ is a dense ideal. If $E \in \mathfrak{B}$, then it bounds the carrier projection of every vector in its range. By Zorn's lemma we can find a maximal family of disjoint carrier projections $E_{\gamma}$ bounded by $E$. If the projection $E-V_{\gamma} E_{\gamma}$ is not zero it bounds a carrier projection, so the maximality of the family implies $E=\bigvee E_{\gamma}$. Moreover, each $E_{\gamma}$ is in $\mathbb{S}$ as we have just shown.

Finally it must be shown that each $E \in \mathbb{C}$ is the carrier projection of a vector. Since $E \in \mathbb{C}$ we may express $E$ is the union of a sequence of disjoint projections $E_{n}$ each of which is the carrier of a vector $x_{n}$ with $\left|x_{n}\right| \leqq 1$. Define $x_{0}=\sum_{n=1}^{\infty} 2^{-n} x_{n}$. Clearly $E x_{0}=x_{0}$. We assert $E$ is the carrier of $x_{0}$. For suppose that $F \in \mathfrak{B}$ and $F x_{0}=x_{0}$. Then for each $n$,

$$
2^{-n} x_{n}=E_{n} x_{0}=E_{n} F x_{0}=F E_{n} x_{0}=2^{-n} F x_{n} .
$$

Thus $F x_{n}=x_{n}, n=1,2, \cdots$, showing $F \geqq E_{n}, n=1,2, \cdots$. It follows that $F \geqq E$, and the proof is complete.

3.2. Definition. If $E \in \mathbb{C}$ we define $m(E)$, the multiplicity of $E$, to be the smallest cardinal power of a set $A$ of vectors such that

$$
E \mathfrak{X}=\operatorname{clm}\{\mathfrak{M}(x) \mid x \in A\} .
$$

3.3. Lemma. If $E, F \in \mathfrak{C}$ and $F \leqq E$, then $m(F) \leqq m(E)$. If $\left\{E_{\alpha}\right\} \subseteq \subseteq$ and $E_{0}=\bigvee E_{\alpha} \in \mathbb{C}$, then $m\left(E_{0}\right)=\bigvee_{\alpha} m\left(E_{\alpha}\right)$. 
Proof. The first statement is clear since if $E \mathfrak{X}=\operatorname{clm}\{\mathfrak{M}(x) \mid x \in A\}$, then $F \mathfrak{X}=\operatorname{clm}\{\mathfrak{M}(F x) \mid x \in A\}$. It follows that $\mathrm{V}_{\alpha} m\left(E_{\alpha}\right) \leqq m\left(E_{0}\right)$. We now observe that there exists a sequence $\left\{G_{k}\right\}$ of disjoint elements in 5 such that each $G_{k}$ is bounded by some $E_{\alpha_{k}}$ and $E_{0}=\bigvee_{k=1}^{\infty} G_{k}$. For we may consider families of disjoint projections in $\mathscr{B}$ each member of which is bounded by an $E_{\alpha}$, and order these families by inclusion. By Zorn's lemma there exists a maximal family which must be countable, since $E_{0} \in \mathbb{S}$. Clearly the union of this maximal family is $E_{0}$. Now let $n_{0}=\mathrm{V} m\left(E_{\alpha}\right)$. There exist families of vectors $x_{n}^{\mathbf{k}}, k=1,2, \cdots$, (some of whose members may be zero) such that $\left|x_{n}^{k}\right| \leqq 1, G_{k} x_{n}^{k}=x_{n}^{k}$, and

$$
G_{k} \mathfrak{X}=\operatorname{clm}\left\{\mathfrak{M}\left(x_{n}^{k}\right) \mid 1 \leqq n \leqq n_{0}\right\}, \quad k=1,2, \cdots .
$$

For each cardinal $n \leqq n_{0}$ define $y_{n}=\sum_{k=1}^{\infty} 2^{-k} x_{n}^{k}$. Then $G_{k} y_{n}=2^{-k} x_{n}^{k}$ and

$$
\begin{aligned}
E_{0} \mathfrak{X} & =\operatorname{clm}\left\{G_{k} \mathfrak{X} \mid k=1,2, \cdots\right\}=\operatorname{clm}\left\{\mathfrak{M}\left(x_{n}^{k}\right) \mid 1 \leqq k<\infty, n \leqq n_{0}\right\} \\
& =\operatorname{clm}\left\{\mathfrak{M}\left(y_{n}\right) \mid n \leqq n_{0}\right\} .
\end{aligned}
$$

Thus $m\left(E_{0}\right) \leqq n_{0}$, showing $m\left(E_{0}\right)=\bigvee m\left(E_{\alpha}\right)$.

3.4. Theorem. Let $\mathfrak{B}$ be a complete B.A. of projections in $\mathfrak{X}$. There exists a unique multiplicity function $m$ defined on $\mathfrak{B}$ with the property that for each $E$ in $\mathfrak{B}$ satisfying the countable chain condition, $m(E)$ is the least cardinal power of a set of cyclic subspaces spanning the range of $E$. There is a unique decomposition of the identity $I=\bigvee E_{n}$ into disjoint projections such that if $E_{n} \neq 0, E_{n}$ has uniform multiplicity $n$.

Proof. This theorem follows immediately from the preceding lemma, Lemma 2.2 and Theorem 2.3.

4. Structure of cyclic subspaces and the separation theorem. Suppose for the moment that $\mathfrak{X}$ is a Hilbert space and $\mathfrak{B}$ is a complete $B$.A. of self-adjoint projections. It will be convenient to regard $\mathscr{B}$ as a spectral measure on the Borel sets $\Sigma$ of its Stone space $\Omega$. We recall that if $f$ is a Borel measurable complex function, the (in general unbounded) normal operator $S(f)=\int_{\Omega} f(\omega) E(d \omega)$ has domain

$$
D(S(f))=\left\{\left.y\left|\int_{\Omega}\right| f(\omega)\right|^{2}(E(d \omega) y, y)<\infty\right\} .
$$

If $x \in \mathfrak{X}$, then $\mathfrak{M}(x)=\{S(f) x \mid x \in D(S(f))\}$. In fact the correspondence $S(f) x$ $\rightarrow f$ defines an isometric isomorphism of the cyclic subspace $\mathfrak{M}(x)$ onto $L_{2}(\Omega, \Sigma, \mu)$, (where $\mu=(E(\cdot) x, x)$ ), and operators of the form $S(g)$ on $\mathfrak{M}(x)$ act under this isomorphism as multiplication by $g$ in $L_{2}(\Omega, \Sigma, \mu)$. If a projection in $\mathfrak{B}$ satisfies the countable chain condition and has multiplicity $n$, we may span its range by $n$ orthogonal cyclic subspaces and the isomorphism extends to an isometric mapping of $\sum_{i \leq n} \mathfrak{M}\left(x_{i}\right)$ onto $\sum_{i \leq n} L_{2}\left(\Omega, \Sigma, \mu_{i}\right)$, 
$\mu_{i}=\left(E(\cdot) x_{i}, x_{i}\right)$. Our aim is to recapture as much as possible of this structure when $\mathfrak{X}$ is an arbitrary Banach space. The main obstacle is the lack of orthogonal complementation, and one must supply a substitute for the measures $(E(\cdot) x, x)$. A suitable representation for the range of $E$ will be obtained only in the case $m(E)<\infty$. However, there the similarity with the Hilbert space case is striking.

Our first objective will be to obtain a characterization of cyclic subspaces similar to that in Hilbert space. Hereafter $\mathfrak{B}$ will be a complete B.A. of projections in a Banach space $\mathfrak{X}$. Regarding $\mathfrak{B}$ as a spectral measure we recall (cf. [7, p. 340]) that for any bounded Borel function $f$, the integral $S(f)$ $=\int \Omega f(\omega) E(d \omega)$ exists in the uniform operator topology and the correspondence $f \rightarrow S(f)$ determines a homomorphism of the algebra of bounded Borel functions on $\Omega$ into the algebra of bounded operators on $\mathfrak{X}$. When $f$ is unbounded we define the sets $e_{m}=\{\omega|| f(\omega) \mid \leqq m\}, m=1,2, \cdots$. The operator $S(f)$ has domain

$$
D(S(f))=\left\{y \mid \lim _{m \rightarrow \infty} \int_{e_{m}} f(\omega) E(d \omega) y \text { exists }\right\}
$$

and is given by the formula

$$
S(f) y=\lim _{m \rightarrow \infty} \int_{e_{m}} f(\omega) E(d \omega) y, \quad y \in D(S(f)) .
$$

In [1] an operational calculus was developed from this definition in the special case that $E(\cdot)$ was a spectral measure on the Borel sets of the complex plane. However, the arguments in [1], with suitable modifications, suffice to prove the properties of the correspondence $f \rightarrow S(f)$ which are collected in the lemma below. A complete discussion will appear in [8, Chapter XVIII].

4.1. Lemma. Let $E(\cdot)$ be the strongly countable additive spectral measure on the Borel sets of $\Omega$ determined by $\mathfrak{B}$. The operator $S(f)$ corresponding to a Borel function is a closed operator with dense domain. Moreover,

(a) $D(S(f))=D(S(|f(\cdot)|)$,

(b) $D(S(f)) \subseteq D(S(g))$ if $|f(\omega)| \geqq|g(\omega)|$,

(c) $S(f)$ is bounded if $f$ is bounded, and

$$
\sup _{\omega \in \Omega}|f(\omega)| \leqq|S(f)| \leqq 4 M \sup _{\omega \in \Omega}|f(\omega)|,
$$

(d) $S(\alpha f)=\alpha S(f)$,

(e) $S(f+g) \supseteq S(f)+S(g)$,

(f) $S(f g) \supseteq S(f) S(g) ; D(S(f) S(g))=D(S(f g)) \cap D(S(g))$,

(g) $S(f) E(e) \supseteq E(e) S(f)$ for $e \in \Sigma$,

(h) $S(f)$ has an inverse if and only if $E\left(f^{-1}(0)\right)=0$, in which case $S(f)^{-1}$ $=S(1 / f)$,

(i) $x^{*} S(f) x=\int_{\Omega} f(\omega) x^{*} E(d \omega) x, x \in D(S(f)), x^{*} \in \mathfrak{X}^{*}$. 
If $x \in \mathfrak{X}$, it is clear that $\mathfrak{M}(x) \supseteq\{S(f) x \mid x \in D(S(f))\}$. A certain amount of machinery will be required to prove that this inclusion is an equality. The fundamental tool is the separation theorem 4.3 proved below. For its proof we shall need some properties of the family $\mathfrak{B}^{*}$ of adjoints of elements of $\mathfrak{B}$. If $E \in \mathfrak{B}$, then $E^{*}$ is a projection in $\mathfrak{X}^{*}$ whose range is the $\mathfrak{X}$-closed manifold $\left\{x^{*} \mid x^{*}(I-E) \mathfrak{X}=0\right\}$. Since $E \bigvee F=E+F-E F$ and $E \wedge F=E F$ in $\mathfrak{B}$ it follows that $(E \bigvee F)^{*}=E^{*} \bigvee F^{*}$ and $(E \wedge F)^{*}=E^{*} \wedge F^{*}$. Also $E^{*} \leqq F^{*}$ if and only if $E \leqq F$ in $\mathfrak{B}$. It follows that $\mathfrak{B}^{*}$ is a $\mathrm{B}$.A. of projections with the same bound as $\mathfrak{B}$. Completeness for $\mathfrak{B}$ implies $\mathfrak{B}^{*}$ is complete as an abstract B.A. and the range of $\bigvee E_{\alpha}^{*}$ is the span of the manifolds $E_{\alpha}^{*} \mathfrak{X}^{*}$ in the $\mathfrak{X}$-topology, while $\left(\Lambda E_{\alpha}^{*}\right) \mathfrak{X}^{*}=\cap E_{\alpha}^{*} \mathfrak{X}^{*}$. These facts are proved in $[3$, p. 348].

4.2. Definition. A closed linear manifold $\mathfrak{M}$ in $\mathfrak{X}$ is called an invariant subspace if $S(f) x \in \mathfrak{M}$ whenever $x \in \mathfrak{M}$ and $x \in D(S(f))$.

4.3. Theorem. Let $\mathfrak{M}$ be a closed invariant subspace in $\mathfrak{X}$ and $x_{0} \in \mathfrak{X}$ with $\mathfrak{M}\left(x_{0}\right) \cap \mathfrak{M}=(0)$. There exists a functional $x_{0}^{*} \in \mathfrak{X}^{*}$ with the properties

(1) $x_{0}^{*}(\mathfrak{M})=(0)$,

(2) $x_{0}^{*} S(f) x_{0}>0$ if $f$ is a non-negative bounded Borel function for which $S(f) x_{0} \neq 0$.

Proof. It is clear that without loss of generality we may suppose the carrier projection of $x_{0}$ (cf. §3) is the identity $I$. Thus if $0 \neq E \in \mathscr{B}$, we have $E x_{0} \neq 0$, and $I$ satisfies the countable chain condition. It will be convenient to isolate a portion of the argument.

Proposition. If $0 \neq F \in \mathfrak{B}$ there exists a projection $G$ in $\mathfrak{B}$ and a nonzero functional $y^{*} \in \mathfrak{X}^{*}$ such that

(i) $G=\Lambda\left\{E \mid y^{*} E x=y^{*} x, x \in \mathfrak{M}\left(x_{0}\right)\right\}$,

(ii) $y^{*}(\mathfrak{M})=(0)$, and

(iii) $0 \neq G \leqq F$.

Proof of the Proposition. Let $z^{*} \in \mathfrak{X}^{*}$ be chosen so that $z^{*}(\mathfrak{M})=(0)$, while $z^{*}\left(F x_{0}\right) \neq 0$. Let $\left.\mathfrak{B}\right)=\left\{E \in \mathfrak{B} \mid z^{*} E x=z^{*} F x, x \in \mathfrak{M}\left(x_{0}\right)\right\}$. Now if $E_{1}, E_{2} \in(\mathfrak{B}$, $z^{*} E_{1} E_{2} x=z^{*} F E_{2} x=z^{*} E_{2} F x=z^{*} F^{2} x=z^{*} F x, x \in \mathfrak{M}\left(x_{0}\right)$, so BS is closed under finite intersections. Thus (S) is a decreasing net when its members are directed by inclusion and therefore converges strongly to the projection

$$
G=\Lambda\{E \mid E \in(B)\}
$$

(cf. §1). Consequently

$$
G^{*} z^{*} x=z^{*} G x=\lim _{E \in \Theta} z^{*} E x=z^{*} F x, \quad x \in \mathfrak{M}\left(x_{0}\right),
$$

and

$$
G^{*} z^{*} y=\lim _{E \in \Theta} z^{*} E y=0, \quad y \in \mathfrak{M} .
$$


If we set $y^{*}=G^{*} z^{*}$, then clearly $G$ and $y^{*}$ satisfy (ii) and (iii). Let $H$ $=\Lambda\left\{E \mid y^{*} E x=y^{*} x, x \in \mathfrak{M}\left(x_{0}\right)\right\}$. Clearly $G \geqq H$. However, if $y^{*} E x=y^{*} x$ for all $x \in \mathfrak{M}\left(x_{0}\right)$, then

$$
\begin{aligned}
z^{*} G E x & =y^{*} E x=y^{*} x=F^{*} G^{*} z^{*} x \\
& =G^{*} z^{*} F x=z^{*} F x,
\end{aligned}
$$

showing $G E \geqq G$. Thus $G \leqq E$, from which it follows $G \leqq H$. Hence $G=H$ and the proof of the Proposition is completed.

Returning now to the proof of Theorem 4.3, let us call a projection $G^{*} \in \mathfrak{B}^{*}$ the $x_{0}$-carrier of the functional $y^{*}$ if condition (i) of the Proposition is satisfied. The Proposition shows the existence of families of disjoint projections in $\mathfrak{B}^{*}$, each of whose members is the $x_{0}$-carrier of a nonzero functional which vanishes on $\mathfrak{M}$. By Zorn's lemma there exists a maximal family $\mathfrak{F}$ of such disjoint projections. Since $I \in \mathfrak{C}$, the family $\mathfrak{F}$ is countable. Let $\mathfrak{F}=\left\{G_{n}^{*}\right\}$ where $G_{n}^{*}$ is the $x_{0}$-carrier of $y_{n}^{*}$. Clearly $\vee G_{n}^{*}=I^{*}$, since otherwise the Proposition, applied to $I-\vee G_{n}$, would contradict the maximality of $\mathfrak{F}$. We may suppose $\sum_{n=1}^{\infty} y_{n}^{*}$ converges and set $y_{0}^{*}=\sum_{n=1}^{\infty} y_{n}^{*}$. We assert that $I^{*}$ is the $x_{0}$-carrier of $y_{0}^{*}$. For if $y_{0}^{*} E x=y_{0}^{*} x, x \in \mathfrak{M}\left(x_{0}\right)$, then for each $n$,

$$
\begin{aligned}
& y_{n}{ }^{*} E x=G_{n}^{*} y_{0}{ }^{*} E x=y_{0}{ }^{*} E G_{n} x \\
& =y_{0}{ }^{*} G_{n} x=y_{n}^{*} x, \quad x \in \mathfrak{M}\left(x_{0}\right),
\end{aligned}
$$

so $E^{*} \geqq G_{n}^{*}, n=1,2, \cdots$.

Finally we obtain $x_{0}^{*}$ from $y_{0}^{*}$. It is convenient again to regard $\mathscr{B}$ as a spectral measure. Let $\nu$ denote the total variation of the measure $y_{0}^{*} E(\cdot) x_{0}$. Then there exists a function $g$ in $L_{1}(\Omega, \Sigma, \nu)$ such that

$$
\nu(e)=\int_{e} g(\omega) y_{0}{ }^{*} E(d \omega) x_{0}, \quad \quad e \in \Sigma .
$$

Thus

$$
\nu(e)=\int_{e}|g(\omega)| \nu(d \omega),
$$

showing $|g(\omega)|=1$, except possibly on a $\nu$-null set. Redefining $g$ to be zero on this set we form $S(g)=\int_{\Omega} g(\omega) E(d \omega)$ and define $x_{0}^{*}=S(g)^{*} y_{0}^{*}$. Thus $x_{0}^{*} E(\cdot) x_{0}$ $=\nu(\cdot)$, and $x_{0}^{*}(\mathfrak{M})=y_{0}^{*}(S(g) \mathfrak{M}) \subseteq y_{0}^{*}(\mathfrak{M})=(0)$. Suppose now that $f$ is a bounded non-negative Borel function and that $x_{0}^{*} S(f) x_{0}=\int_{\Omega} f(\omega) x_{0}^{*} E(d \omega) x_{0}$ $=0$. Clearly then

$$
y_{0}^{*} E(e) x_{0}=0, \quad e \in \Sigma, \quad e \subseteq e_{0},
$$

where $e_{0}=\{\omega \mid f(\omega)>0\}$. Thus $y_{0}^{*}\left(\mathfrak{M}\left(E\left(e_{0}\right) x_{0}\right)\right)=(0)$, so $y_{0}^{*}\left(I-E\left(e_{0}\right)\right) x=y_{0}^{*} x$, $x \in \mathfrak{M}\left(x_{0}\right)$. Since $I^{*}$ is the $x_{0}$-carrier of $y_{0}^{*}$ we must have $E\left(e_{0}\right)=0$, showing $S(f) x_{0}=0$. 
4.4. Corollary. Let $\mathfrak{B}$ be a complete B.A. of projections in $\mathfrak{X}$ and let $x_{0} \in \mathfrak{X}$. There exists a functional $x_{0}^{*}$ in $\mathfrak{X}^{*}$ such that the measure $x_{0}^{*} E(\cdot) x_{0}$ is positive and dominates the vector measure $E(\cdot) x_{0}$.

4.5. Theorem. If $x_{0} \in \mathfrak{X}$, then $\mathfrak{M}\left(x_{0}\right)=\left\{S(f) x_{0} \mid x_{0} \in D(S(f))\right\}$.

Proof. Let $x_{0}^{*}$ be chosen as in Corollary 4.4 and let $\mu_{0}=x_{0}^{*} E(\cdot) x_{0}$. Now $\mu_{0}(e)=0$ implies $E(d) x_{0}=0$ for $d \subseteq e$, and thus that $\mathfrak{M}\left(E(e) x_{0}\right)=(0)$. Thus if $x \in \mathfrak{M}\left(x_{0}\right), x_{0}^{*} E(e) x=0$. By the Radon-Nikodym theorem there exists a function $f \in L_{1}\left(\Omega, \Sigma, \mu_{0}\right)$ such that

$$
x_{0}^{*} E(e) x=\int_{e} f(\omega) \mu_{0}(d \omega), \quad e \in \Sigma .
$$

The correspondence $T: x \rightarrow f$ defines a linear map of $\mathfrak{M}\left(x_{0}\right)$ into $L_{1}\left(\Omega, \Sigma, \mu_{0}\right)$. It is continuous since $\int_{\Omega}|f(\omega)| \mu_{0}(d \omega)=$ tot. var. $x_{0}^{*} E(\cdot) x \leqq 4 M\left|x_{0}^{*}\right||x|$. For each $m$ let $e_{m}=\{\omega|| f(\omega) \mid \leqq m\}$. We shall show that $E\left(e_{m}\right) x=S\left(f \cdot k_{e_{m}}\right) x_{0}$, the symbol $k_{e}$ denoting the characteristic function of the set $e$. If $x^{*} \in \mathfrak{X}^{*}$, then $x^{*} E(\cdot) x_{0}$ is dominated by $\mu_{0}$, so

$$
x^{*} E(e) x_{0}=\int_{e} p(\omega) \mu_{0}(d \omega),
$$$$
e \in \Sigma
$$

where $p \in L_{1}\left(\Omega, \Sigma, \mu_{0}\right)$. Let $d$ be a subset of $e_{m}$ on which $p$ is bounded, and let $\left\{g_{n}\right\}$ be a sequence of bounded functions such that $x=\lim S\left(g_{n}\right) x_{0}$. Then

$$
\begin{aligned}
x^{*} S\left(g_{n}\right) E(d) x_{0} & =\int_{d} g_{n}(\omega) p(\omega) \mu_{0}(d \omega) \rightarrow \int_{d} f(\omega) p(\omega) \mu_{0}(d \omega) \\
& =x^{*} S\left(f \cdot k_{d}\right) x_{0},
\end{aligned}
$$

by continuity of $T$. However $x^{*} S\left(g_{n}\right) E(d) x_{0} \rightarrow x^{*} E(d) x$. Thus $x^{*} S\left(f \cdot k_{d}\right) x_{0}$ $=x^{*} E(d) x$. By a limiting argument $x^{*} S\left(f \cdot k_{e_{m}}\right) x_{0}=x^{*} E\left(e_{m}\right) x$. However, $x^{*}$ is arbitrary so $E\left(e_{m}\right) x=S\left(f \cdot k_{e_{m}}\right) x_{0}$. Since $x=\lim E\left(e_{m}\right) x, x_{0} \in D(S(f))$ and $x=S(f) x_{0}$.

4.6. Corollary. If $f$ is a Borel function and $x_{0} \in D(S(f))$, then $f \in L_{1}\left(\Omega, \Sigma, x_{0}^{*} E(\cdot) x_{0}\right)$ for every $x_{0}^{*}$ satisfying the conditions of Corollary 4.4.

We close this section with a useful lemma.

4.7. Lemma. Let $\mathfrak{M}$ be a closed invariant subspace and let $y_{0} \in \mathfrak{X}$. There exists a maximal projection $E_{0} \in \mathfrak{B}$ such that $E_{0} y_{0} \in \mathfrak{M}$. Moreover,

$$
\mathfrak{M} \cap \mathfrak{M}\left(\left(I-E_{0}\right) y_{0}\right)=(0) .
$$

Proof. If $z \in \mathfrak{M}\left(y_{0}\right) \cap \mathfrak{M}$ then, by Theorem $4.5, z=S(f) y_{0}$ for some Borel function $f$. If $e$ is any set in $\Sigma$ on which $f$ satisfies an inequality $K^{-1} \leqq|f(\omega)|$ $\leqq K$ then $E(e) y_{0}=S(g) z$, where $g=f^{-1} k_{e}$. Thus if $\mathfrak{M}\left(y_{0}\right) \cap \mathfrak{M} \neq(0)$, there exist projections $E$ with $0 \neq E y_{0} \in \mathfrak{M}\left(y_{0}\right) \cap \mathfrak{M}$. Let $E_{0}$ be the supremum of such 
projections. If $\mathfrak{M}\left(\left(I-E_{0}\right) y_{0}\right) \cap \mathfrak{M} \neq(0)$, the same argument may be used to contradict the maximality of $E_{0}$. If $\mathfrak{M}\left(y_{0}\right) \cap \mathfrak{M}=(0)$, then $I-E_{0}$ is the carrier projection of $y_{0}$ (cf. $\S 3$ ).

5. Representation of EX. In this section we shall examine the range of a projection $E$ of finite uniform multiplicity. Since every projection in $\mathbb{B}$ is the union of disjoint projections in $\mathbb{S}$ it will be sufficient to suppose $E \in \mathbb{C}$. To avoid notational difficulties we shall suppose the identity is in $\mathbb{E}$ and has finite uniform multiplicity $n$. Then there exist vectors $x_{1}, \cdots, x_{n}$ such that ${ }^{3}$ ) $\mathfrak{X}=\bigvee_{i=1}^{n} \mathfrak{M}\left(x_{i}\right)$ and no smaller number of cyclic subspaces will span $\mathfrak{X}$. We shall see that the manifolds $\mathfrak{M}\left(x_{i}\right)$ for such a minimal spanning set are all disjoint and that we may imbed $\mathfrak{X}$ in a direct sum of $L_{1}$ spaces $\sum_{i=1}^{n} L_{1}\left(\Omega, \Sigma, \mu_{i}\right)$ analogous to the imbedding in a sum of $L_{2}$ spaces in the classical Hilbert space case. The measures $\mu_{i}$ have the form $x_{i}^{*} E(\cdot) x_{i} ; x_{i}^{*} \in \mathfrak{X}^{*}$. This fact is the key to the later discussion of the duality between multiplicity in $\mathfrak{X}$ and in $\mathfrak{X}^{*}$.

5.1. Lemma. Let the identity satisfy the countable chain condition and have finite uniform multiplicity $n$. If $x_{1}, \cdots, x_{n}$ is any set of vectors such that $\mathfrak{X}=\mathrm{V}_{i=1}^{n} \mathfrak{M}\left(x_{i}\right)$, then

(1) the carrier of each $x_{i}$ is $I$,

(2) $\mathfrak{M}\left(x_{i}\right) \cap \bigvee_{j \neq i} \mathfrak{M}\left(x_{j}\right)=(0), i=1, \cdots, n$.

(3) For each $i$ there exists a linear functional $x_{i}^{*}$ such that $x_{i}^{*}\left(\bigvee_{j \neq i} \mathfrak{M}\left(x_{j}\right)\right)$ $=(0)$, while $x_{i}^{*} S(f) x_{i}>0$ for each non-negative Borel function $f$ such that $S(f) x_{i} \neq 0$.

Proof. To prove (2) suppose, for example, that there is a nonzero vector $z \in \mathfrak{M}\left(x_{n}\right) \cap \bigvee_{i=1}^{n-1} \mathfrak{M}\left(x_{i}\right)$. By Lemma 4.7 we can find a projection $E$ with $0 \neq E x_{n} \in \bigvee_{i=1}^{n-1} \mathfrak{M}\left(x_{i}\right)$. Hence $E \mathfrak{X}=\bigvee_{i=1}^{n-1} \mathfrak{M}\left(E x_{i}\right)$ which shows $m(E) \leqq n-1$ and contradicts the assumption of uniform multiplicity. Statement (1) is proved in a similar way, for if $E$ is the carrier of $x_{i}$ then $(I-E) \mathfrak{X} \subseteq \bigvee_{j \neq i} \mathfrak{M}\left((I-E) x_{j}\right)$. Statement (3) follows from Theorem 4.3.

5.2. Theorem. Let the hypotheses of Lemma 5.1 be satisfied. There exists a linear continuous one to one map $T$ of $\mathfrak{X}$ onto a dense subspace of the direct sum $\sum_{i=1}^{n} L_{1}\left(\Omega, \Sigma, \mu_{i}\right), \mu_{i}=x_{i}^{*} E(\cdot) x_{i}$; such that if $T x=\left[f_{1}, \cdots, f_{n}\right]$ then

(a) $x_{i}^{*} E(e) x=\int_{e} f_{i}(\omega) \mu_{i}(d \omega), \quad e \in \Sigma, i=1, \cdots, n$.

(b) $x=\lim _{m \rightarrow \infty} \sum_{i=1}^{n} S\left(f_{i} \cdot k_{e_{m}}\right) x_{i}$ where

$$
e_{m}=\left\{\omega|| f_{i}(\omega) \mid \leqq m, i=1, \cdots, n\right\} .
$$

Proof. The proof proceeds very much like that of Theorem 4.5. Given $x \in \mathfrak{X}$, there exist bounded functions $g_{i}^{m}$ such that

$$
x=\lim _{m \rightarrow \infty} \sum_{i=1}^{n} S\left(g_{i}^{m}\right) x_{i} .
$$

(3) It is convenient to denote by $\bigvee_{i-1}^{n} \mathfrak{M}\left(x_{i}\right)$ the smallest closed linear manifold containing the manifolds $\mathfrak{M}\left(x_{i}\right), i=1, \cdots, n$. 
For each $e \in \Sigma$

$$
x_{i}^{*} E(e) x=\lim _{m \rightarrow \infty} x_{i}^{*} S\left(g_{i}^{m} \cdot k_{e}\right) x_{i} .
$$

Thus $\mu_{i}(e)=0$ implies $E(d) x_{i}=0, d \subseteq e$, so $x_{i}^{*} E(e) x=0$. Consequently, for each $i$ there is a function $f_{i} \in L_{1}\left(\Omega, \Sigma, \mu_{i}\right)$ such that

$$
x_{i}^{*} E(e) x=\int_{e} f_{i}(\omega) \mu_{i}(d \omega) .
$$

Since

$$
\begin{aligned}
\sup _{i} \int_{\Omega}\left|f_{i}(\omega)\right| \mu_{i}(d \omega) & =\sup _{i} \text { tot. var. }\left\{x_{i}^{*} E(\cdot) x\right\} \\
& \leqq 4 M|x| \sup _{i}\left|x_{i}^{*}\right|
\end{aligned}
$$

the linear map $T: x \rightarrow\left[f_{1}, \cdots, f_{n}\right]$ is a continuous map of $\mathfrak{X}$ into $\sum_{i=1}^{n} L_{1}\left(\Omega, \Sigma, \mu_{i}\right)$. Now let $e_{m}=\left\{\omega|| f_{i}(\omega) \mid \leqq m, i=1, \cdots, n\right\}$. We show next that

$$
E\left(e_{m}\right) x=\sum_{i=1}^{n} S\left(f_{i} \cdot k_{e_{m}}\right) x_{i}, \quad m=1,2, \cdots
$$

Fix $m$ and let $x^{*} \in \mathfrak{X}^{*}$. We may find functions $p_{i} \in L_{1}\left(\Omega, \Sigma, \mu_{i}\right)$ such that

$$
x^{*} E(e) x_{i}=\int_{e} p_{i}(\omega) \mu_{i}(d \omega),
$$

If $d$ is an arbitrary subset of $e_{m}$ on which $p_{1}, \cdots, p_{n}$ are all bounded

$$
\begin{aligned}
\sum_{i=1}^{n} x^{*} S\left(g_{i}^{k}\right) E(d) x_{i} & =\sum_{i=1}^{n} \int_{d} g_{i}^{k}(\omega) p_{i}(\omega) \mu_{i}(d \omega) \\
& \rightarrow \sum_{i=1}^{n} \int_{d} f_{i}(\omega) p_{i}(\omega) \mu_{i}(d \omega) \\
& =\sum_{i=1}^{n} x^{*} S\left(f_{i} \cdot k_{d}\right) x_{i}
\end{aligned}
$$

However also

$$
\lim _{k \rightarrow \infty} \sum_{i=1}^{n} x^{*} S\left(g_{i}^{k}\right) E(d) x_{i}=x^{*} E(d) x .
$$

A limiting argument shows

$$
x^{*} E\left(e_{m}\right) x=\sum_{i=1}^{n} x^{*} S\left(f_{i} \cdot k_{e_{m}}\right) x_{i} .
$$


Since $x^{*}$ is arbitrary we have proved $\left({ }^{*}\right)$. Statement (b) is obtained by letting $m \rightarrow \infty$. Also (b) shows that if $T x=0$, then $x=0$. The range of $T$ is dense as it contains all $n$-tuples of bounded functions.

5.3. CoROllary. Under the hypotheses of Theorem 5.2 [ $\left.\mathrm{V}_{i \in \sigma} \mathfrak{M}\left(x_{i}\right)\right]$ $\cap\left[\bigvee_{j \in \sigma^{\prime}} \mathfrak{M}\left(x_{j}\right)\right]=(0)$ for any partition $\left[\sigma, \sigma^{\prime}\right]$ of the set $[1, \cdots, n]$.

Proof. Suppose for example that $0 \neq z \in V_{i=1}^{p} \mathfrak{M}\left(x_{i}\right) \cap V_{i=p+1}^{n} \mathfrak{M}\left(x_{i}\right)$. Then there exists a set $e \in \Sigma$ and bounded functions $g_{i}$ such that

$$
E(e) z=\sum_{i=1}^{n} S\left(g_{i}\right) E(e) x_{i}, \quad E(e) z \neq 0 .
$$

Suppose without loss of generality that $S\left(g_{n}\right) E(e) x_{n} \neq 0$. Then it belongs to $\bigvee_{i=1}^{n-1} \mathfrak{M}\left(x_{i}\right)$, contradicting the fact $\mathfrak{M}\left(x_{n}\right) \cap \bigvee_{i=1}^{n-1} \mathfrak{M}\left(x_{i}\right)=(0)$.

REMARK. It is interesting to note in connection with Lemma 5.1 that $\mathfrak{X}$ is not in general the algebraic direct sum of the manifolds $\mathfrak{M}\left(x_{i}\right)$. In fact, Dieudonné [5] has constructed an ingenious example of a space $\mathfrak{X}$ and B.A. of projections such that every nonzero $E \in \mathfrak{B}$ has multiplicity two. However, for no choice of $x_{1}$ and $x_{2}$ or $E \in \mathfrak{B}$ is $E \mathfrak{X}$ the algebraic direct sum of $\mathfrak{M}\left(E x_{1}\right)$ and $\mathfrak{M}\left(E x_{2}\right)$.

6. The multiplicity function for $\mathfrak{B}^{*}$. The next three sections are devoted to defining a multiplicity function on the adjoint B.A. $\mathfrak{B}^{*}$ in $\mathfrak{X}^{*}$ and proving theorems analogous to those established for $\mathfrak{B}$. The relevant topology in $\mathfrak{X}^{*}$ is now the weak ${ }^{*}$ or $\mathfrak{X}$-topology. The range of a projection in $\mathfrak{B}^{*}$ is an $\mathfrak{X}$ closed manifold. If $\left\{E_{\alpha}\right\} \subseteq \mathscr{B}$ then $\left(\vee E_{\alpha}^{*}\right) \mathfrak{X}^{*}$ is the span of the manifolds $E_{\alpha}^{*} \mathfrak{X}^{*}$ in the $\mathfrak{X}$-topology and $\left(\Lambda E_{\alpha}^{*}\right) \mathfrak{X}^{*}=\cap E_{\alpha}^{*} \mathfrak{X}^{*}$. Moreover if $\left\{E_{\alpha}^{*}\right\}$ is an increasing (decreasing) net in $\mathfrak{B}^{*}, \lim E_{\alpha}^{*} x^{*} x=\left(\bigvee E_{\alpha}^{*}\right) x^{*} x\left(\wedge E_{\alpha}^{*} x^{*} x\right)$ for each $x \in \mathfrak{X}, x^{*} \in \mathfrak{X}^{*}$. These facts are proved in [3].

For a given $x^{*} \in \mathfrak{X}^{*}$ we define $\mathfrak{R}\left(x^{*}\right)$ to be the span in the $\mathfrak{X}$-topology of the set of vectors $\left\{E^{*} x^{*} \mid E^{*} \in \mathfrak{B}^{*}\right\}$; and call it the cyclic subspace generated by $x^{*}$. These subspaces will be the building blocks for the multiplicity theory in $\mathfrak{X}^{*}$. The symbol $\mathfrak{S}^{*}$ will denote the family of projections in $\mathfrak{B}^{*}$ which satisfy the countable chain condition. Clearly $E^{*} \in \mathfrak{C}^{*}$ if and only if $E \in \mathfrak{C}$. The projection $\Lambda\left\{E^{*} \mid E^{*} x^{*}=x^{*}\right\}$ is called the carrier projection of $x^{*}$.

6.1. Lemma. The family $\mathfrak{S}^{*}$ is a dense $\sigma$-ideal in $\mathfrak{B}^{*}$. Each $E^{*} \in \mathfrak{C}^{*}$ is the carrier projection of a vector in $\mathfrak{X}^{*}$.

Proof. The first statement is clear. To prove the second let $E^{*} \in \mathfrak{C}^{*}$ and recall that $E$ is the carrier of a vector $x_{0} \in \mathfrak{X}$, by Lemma 3.1. Let $x_{0}^{*}$ be chosen as in Corollary 4.4. Replacing $x_{0}^{*}$ if necessary by $E^{*} x_{0}^{*}$, we may suppose $E^{*} x_{0}^{*}=x_{0}^{*}$. Suppose $F^{*} \in B^{*}$ and $F^{*} x_{0}^{*}=x_{0}^{*}$. Then $\left(I^{*}-F^{*}\right) x_{0}^{*}=0$. Thus $x_{0}^{*}(I-F) x_{0}=0$, from which it follows $F x_{0}=x_{0}$. Thus $F \geqq E$ so $F^{*} \geqq E^{*}$, showing $E^{*}$ is the carrier of $x_{0}^{*}$.

Not every carrier projection in $\mathfrak{B}^{*}$ belongs to $\mathfrak{C}^{*}$ as one sees from the 
following example. Let $R$ be the real line, $\Gamma$ be the $\sigma$-field of all subsets of $R$ and $\gamma$ be the measure which assigns to each point in $R$ the mass one. Taking $\mathfrak{X}=L_{1}(R, \Gamma, \gamma), \mathfrak{X}^{*}=M(R)$, the Banach space of all bounded functions on $R$. For $e \in \Gamma$ define $(E(e) f)(r)=\delta_{r}^{e} f(r)$ where $\delta_{r}^{e}=0$ if $r \notin e, \delta_{r}^{e}=1$ if $r \in e$. The B.A. of such projections is complete and $\mathbb{E}$ corresponds to the countable sets. If $x^{*}$ is the function identically one in $M(R)$, its carrier is $I^{*}$ which is not in $\mathfrak{E}^{*}$.

6.2. Definition. If $E^{*} \in \mathfrak{C}^{*}$ we define $m\left(E^{*}\right)$, the multiplicity of $E^{*}$, to be the smallest cardinal power of a set $A$ of vectors such that $E^{*} \mathfrak{X}^{*}$ is spanned in the $\mathfrak{X}$-topology by the manifolds $\left\{\mathfrak{R}\left(x^{*}\right) \mid x^{*} \in A\right\}$.

The next lemma establishes the required continuity properties.

6.3. Lemma. If $E^{*}, F^{*} \in \mathfrak{C}^{*}$ and $F^{*} \leqq E^{*}$, then $m\left(F^{*}\right) \leqq m\left(E^{*}\right)$. If $\left\{E_{\alpha}^{*}\right\}$ $\subseteq \mathfrak{C}^{*}$ and $E_{0}^{*}=\mathrm{V} E_{\alpha}^{*} \in \mathfrak{C}^{*}$, then $m\left(E_{0}^{*}\right)=\bigvee m\left(E_{\alpha}^{*}\right)$.

Proof. The proof is almost identical to that of Lemma 3.3. For example, in the proof of the second statement one shows, using Zorn's lemma as before, that $E_{0}^{*}=\bigvee_{n=1}^{\infty} G_{n}^{*}$, where each $G_{n}^{*}$ belongs to $\mathfrak{\complement}^{*}$ and dominates some $E_{\alpha_{n}}^{*}$. There exist families $\left\{x_{n}^{* k}\right\}$ such that $G_{k}^{*} \mathfrak{X}^{*}$ is spanned in the $\mathfrak{X}$-topology by the manifolds $\left\{\mathfrak{N}\left(x_{n}^{* k}\right) \mid 1 \leqq n \leqq n_{0}\right\}, n_{0}=\mathrm{V}_{m}\left(E_{\alpha}^{*}\right)$. The functionals $y_{n}^{*}=\sum_{k=1}^{\infty} 2^{-k} x_{n}^{* k}$ $\operatorname{span} E_{0}^{*} \mathfrak{X}^{*}$.

Now Theorem 2.3 yields

6.4. THEOREM. There exists a unique multiplicity function $m$ defined on $\mathfrak{B}^{*}$ with the property that for each $F^{*} \in \mathfrak{C}^{*}, m\left(F^{*}\right)$ is the least cardinal power of a set of cyclic subspaces spanning $F^{*} \mathfrak{X}^{*}$ in the $\mathfrak{X}$-topology. There is a unique decomposition of the identity $I^{*}=\bigvee F_{n}^{*}$ into disjoint projections such that if $F_{n}^{*} \neq 0, F_{n}^{*}$ has uniform multiplicity $n$.

7. Cyclic subspaces and separation theorem in $\mathfrak{X}^{*}$. We may also consider $\mathfrak{B}^{*}$ as a projection valued measure on the Borel sets $\Sigma$ of $\Omega$. Now the measures $E^{*}(\cdot) x^{*} x$ are countably additive for each $x \in \mathfrak{X}, x^{*} \in \mathfrak{X}^{*}$. However, the vector measures $E^{*}(\cdot) x^{*}$ may not be countably additive unless $\mathfrak{X}$ is reflexive. If $f$ is a bounded Borel function the integral $S^{*}(f)=\int_{\Omega} f(\omega) E^{*}(d \omega)$ exists in the uniform operator topology and clearly the operator $S^{*}(f)$ is just the adjoint $S(f)^{*}$ of $S(f)=\int_{\Omega} f(\omega) E(d \omega)$. Thus if $f$ is an unbounded function we are led to define $S^{*}(f)$ to be the adjoint of the closed, densely defined operator $S(f)$. That is, $D\left(S^{*}(f)\right)$ is the set of all $x^{*}$ such that $z^{*}(x)=x^{*} S(f) x$ is continuous for $x$ in $D(S(f))$ and $S^{*}(f) x^{*}=z^{*}$ for $x^{*} \in D\left(S^{*}(f)\right)$. The proof of the following lemma is straightforward and will be omitted.

7.1. Lemma. (a) The operator $S^{*}(f)$ is closed and $D\left(S^{*}(f)\right)$ is $\mathfrak{X}$-dense in $\mathfrak{X}^{*}$.

(b) Let $e_{m}=\{\omega|| f(\omega) \mid \leqq m\}$. Then $x^{*} \in D\left(S^{*}(f)\right)$ if and only if

$\lim _{n \rightarrow \infty} S^{*}\left(f \cdot k_{e_{m}}\right) x^{*} x$ exists for each $x \in \mathfrak{X}$.

If this limit exists it equals $S^{*}(f) x^{*} x$. 
(c) If $g$ is a bounded function and $x^{*} \in D\left(S^{*}(f)\right)$, then $S^{*}(g) x^{*} \in D\left(S^{*}(f)\right)$ and $S^{*}(g) S^{*}(f) x^{*}=S^{*}(f) S^{*}(g) x^{*}$.

The next task is to prove that $\mathfrak{R}\left(x^{*}\right)=\left\{S^{*}(f) x^{*} \mid x^{*} \in D\left(S^{*}(f)\right)\right\}$. For this and other purposes we shall need a separation theorem analogous to Theorem 4.3.

7.2. Theorem. Let $\mathfrak{N}$ be an $\mathfrak{X}$-closed invariant subspace in $\mathfrak{X}^{*}$ and let $x_{0}^{*}$ be a functional whose carrier is in $\mathfrak{S}^{*}$. If $\mathfrak{R} \cap \mathfrak{N}\left(x_{0}^{*}\right)=(0)$, then there exists a vector $x_{0} \in \mathfrak{X}$ such that

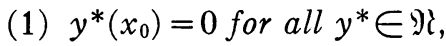

(2) $S^{*}(f) x_{0}^{*} x_{0}>0$ for each bounded non-negative Borel function $f$ such that $S^{*}(f) x_{0}^{*} \neq 0$.

Proof. We shall merely outline the proof as it is similar to that of Theorem 4.3. As before it is sufficient to suppose the carrier of $x_{0}^{*}$ is the identity $I^{*}$. It is well known that the $\mathfrak{X}$-continuous linear functionals on $\mathfrak{X}^{*}$ are precisely those induced by elements of $\mathfrak{X}$. Also one knows (cf. [3]) that if $\left\{G_{\alpha}^{*}\right\}$ is a decreasing net in $\mathfrak{B}^{*}, \lim G_{\alpha}^{*} x^{*}(x)=\left(\wedge G_{\alpha}^{*}\right) x^{*}(x)$ for $x \in \mathfrak{X}, x^{*} \in \mathfrak{X}^{*}$. Using these two facts it follows much as before that if $0 \neq F^{*} \in \mathfrak{B}^{*}$, there exists a projection $G^{*}$ in $\mathfrak{B}^{*}$ and a nonzero vector $y$ in $\mathfrak{X}$ such that

(i) $G^{*}=\Lambda\left\{E^{*} \mid E^{*} x^{*} y=x^{*} y, x^{*} \in \mathfrak{N}\left(x_{0}^{*}\right)\right\}$,

(ii) $y^{*} y=0$ for $y^{*} \in \mathfrak{N}$,

(iii) $0 \neq G^{*} \leqq F^{*}$.

Now using Zorn's lemma express $I^{*}$ as the union of a sequence of disjoint projections $G_{n}^{*}$, each of which satisfies (i) for an appropriate vector $y_{n}$ of norm one. The vector $x_{0}$ is obtained from $y_{0}=\sum_{n=1}^{\infty} 2^{-n} y_{n}$ by the formula $x_{0}=S(f) y_{0}$, where $f$ is the Radon-Nikodym derivative of tot. var. $x_{0}^{*} E(\cdot) y_{0}$ with respect to $x_{0}^{*} E(\cdot) y_{0}$.

\subsection{Theorem. If $x_{0}^{*} \in \mathfrak{X}^{*}$, then}

$$
\Re\left(x_{0}^{*}\right)=\left\{S^{*}(f) x_{0}^{*} \mid x_{0}^{*} \in D\left(S^{*}(f)\right)\right\} .
$$

Proof. It is clear from Lemma 7.1 that each functional $S^{*}(f) x_{0}^{*}$ is in $\Re\left(x_{0}^{*}\right)$. To prove the converse it will be sufficient to suppose the carrier of $x_{0}^{*}$ is in $\mathfrak{C}^{*}$, since in any case it is the union of disjoint projections in $\mathfrak{C}^{*}$. If $z^{*} \in \mathfrak{N}\left(x_{0}^{*}\right)$, there exists a net $\left\{g_{\alpha}\right\}$ of finite linear combinations of characteristic functions such that

$$
S^{*}\left(g_{\alpha}\right) x_{0}^{*}(x) \rightarrow z^{*}(x), \quad x \in \mathfrak{i} .
$$

By Theorem 7.2 there exists a vector $x_{0}$ such that

$$
\nu_{0}=E^{*}(\cdot) x_{0}^{*} x_{0}=x_{0}^{*} E(\cdot) x_{0} \geqq 0,
$$

while if $E^{*}\left(e_{0}\right) x_{0}^{*} x_{0}=0$ then $E^{*}\left(e_{0}\right) x_{0}^{*}=0$. Clearly $z^{*} E(\cdot) x_{0}$ is dominated by $\nu_{0}$ so there exists an $f \in L_{1}\left(\Omega, \Sigma, \nu_{01}\right)$ such that 


$$
z^{*} E(e) x_{0}=\int_{e} f(\omega) \nu_{0}(d \omega), \quad e \in \Sigma .
$$

If $h$ is any bounded Borel function

$$
\begin{aligned}
\int_{\Omega} f(\omega) h(\omega) \nu_{0}(d \omega) & =z^{*} S(h) x_{0} \\
& =\lim _{\alpha} S^{*}\left(g_{\alpha}\right) x_{0}^{*} S(h) x_{0} \\
& =\lim _{\alpha} \int_{\Omega} g_{\alpha}(\omega) h(\omega) \nu_{0}(d \omega),
\end{aligned}
$$

showing $g_{\alpha} \rightarrow f$ weakly in $L_{1}\left(\Omega, \Sigma, \nu_{0}\right)$.

It remains to show that $z^{*}=S^{*}(f) x_{0}^{*}$. If $x \in \mathfrak{X}$, then $\nu_{0}$ dominates $x_{0}^{*} E(\cdot) x$ $=E^{*}(\cdot) x_{0}^{*} x$, since $\nu_{0}(e)=0$ implies $E^{*}(e) x_{0}^{*}=0$, as remarked above. Let $p \in L_{1}\left(\Omega, \Sigma, \nu_{0}\right)$ be chosen so that

$$
x_{0}^{*} E(e) x=\int_{e} p(\omega) \nu_{0}(d \omega), \quad e \in \Sigma,
$$

and set $e_{m}=\{\omega|| f(\omega) \mid \leqq m\}, m=1,2, \cdots$. If $d$ is any subset of $e_{m}$ on which $p$ is bounded,

$$
\begin{aligned}
S^{*}\left(g_{\alpha}\right) x_{0}^{*} E(d) x & =\int_{d} g_{\alpha}(\omega) p(\omega) \nu_{0}(d \omega) \\
& \rightarrow \int_{d} f(\omega) \nu_{0}(d \omega),
\end{aligned}
$$

as $g_{\alpha} \rightarrow f$ weakly. Thus $z^{*} E(d) x=S^{*}\left(f \cdot k_{d}\right) x_{0}^{*} x$. By a limiting argument $z^{*} E\left(e_{m}\right) x=S^{*}\left(f \cdot k_{e_{m}}\right) x_{0}^{*} x$, and since $x$ is arbitrary, $E^{*}\left(e_{m}\right) z^{*}=S^{*}\left(f \cdot k_{e_{m}}\right) x_{0}^{*}$. It now follows easily that $x_{0}^{*} \in D\left(S^{*}(f)\right)$ and $z^{*}=S^{*}(f) x_{0}^{*}$.

Analogous to Lemma 4.7 we have

7.4. LemMa. Let $\mathfrak{R}$ be an $\mathfrak{X}$-closed invariant subspace in $\mathfrak{X} *$ and let $y_{0}^{*} \in \mathfrak{X}^{*}$. There exists a maximal projection $E_{0}^{*} \in \mathfrak{B}^{*}$ such that $E_{0}^{*} y_{0}^{*} \in \mathfrak{\Re}$. Moreover $\mathfrak{N} \cap \mathfrak{N}\left(\left(I^{*}-E_{0}^{*}\right) x_{0}^{*}\right)=(0)$.

The proof is similar and will be omitted.

8. Representation of $E^{*} \mathfrak{X}^{*}$. We turn now to the representation of $E^{*} \mathfrak{X}^{*}$ when $E^{*} \in \mathfrak{C}^{*}$ and has finite uniform multiplicity $n$. The results are parallel to those in $\mathfrak{X}$. The weaker form of completeness enjoyed by $\mathfrak{B}^{*}$ leads to a weaker form of the representation theorem, as the relevant topology in $\mathfrak{X}^{*}$ is now the $\mathfrak{X}$-topology. As before we shall suppose for convenience that $E^{*}$ is the identity $I^{*}$. 
8.1. Lemma. Let the identity $I^{*}$ in $\mathfrak{B}^{*}$ satisfy the countable chain condition and have finite uniform multiplicity $n$. If $x_{1}^{*}, \cdots, x_{n}^{*}$ is any set of functionals such that $\left.{ }^{4}\right) \mathfrak{X}^{*}=\mathrm{V}_{i=1}^{n} \mathfrak{R}\left(x_{i}^{*}\right)$, then

(1) the carrier of each $x_{i}^{*}$ is $I^{*}$.

(2) $\mathfrak{N}\left(x_{i}^{*}\right) \cap \bigvee_{j \neq i} \mathfrak{R}\left(x_{j}^{*}\right)=(0), i=1, \cdots, n$.

(3) For each $i$ there exists a vector $x_{i} \in \mathfrak{X}$ such that $y^{*}\left(x_{i}\right)=0$ for $y^{*} \in \bigvee_{j \neq i} \mathfrak{\Re}\left(x_{j}^{*}\right)$, while $S^{*}(f) x_{i}^{*} x_{i}>0$ for each non-negative bounded Borel function $f$ such that $S^{*}(f) x_{i}^{*} \neq 0$.

Proof. This lemma is proved from Lemma 7.4 and Theorem 7.2 exactly as Lemma 5.1 was proved from Lemma 4.7 and Theorem 4.3.

8.2. Theorem. Let the hypotheses of Lemma 8.1 be satisfied. There exists a linear one to one map $U$ of $\mathfrak{X}^{*}$ onto a (norm) dense subspace of the direct sum $\sum_{i=1}^{n} L_{1}\left(\Omega, \Sigma, \nu_{i}\right), \nu_{i}=E^{*}(\cdot) x_{i}^{*} x_{i} ;$ such that if $U x^{*}=\left[f_{1}, \cdots, f_{n}\right]$ then

(a) $E^{*}(e) x^{*} x_{i}=\int_{e} f_{i}(\omega) \nu_{i}(d \omega), e \in \Sigma, i=1, \cdots, n$.

(b) $x^{*}(x)=\lim _{m \rightarrow \infty} \sum_{i=1}^{n} S^{*}\left(f_{i} \cdot k_{e_{m}}\right) x_{i}^{*}(x), x \in \mathfrak{X}$, where

$$
e_{m}=\left\{\omega|| f_{i}(\omega) \mid \leqq m, i=1, \cdots, n\right\} .
$$

The map $U$ is continuous when $\mathfrak{X}^{*}$ and $\sum_{i=1}^{n} L_{1}\left(\Omega, \Sigma, \nu_{i}\right)$ have their norm topologies and also when $\mathfrak{X}^{*}$ has its $\mathfrak{X}$-topology and $\sum_{i=1}^{n} L_{1}\left(\Omega, \Sigma, \nu_{i}\right)$ has its weak topology.

Proof. Most of the proof of this theorem is a straight-forward modification of the proof of Theorem 5.2. Given $x^{*} \in \mathfrak{X}^{*}$ there exist nets $g_{i}^{\alpha}$ of bounded functions such that

$$
x^{*}(x)=\lim \sum_{i=1}^{n} S^{*}\left(g_{i}^{\alpha}\right) x^{*}(x), \quad x \in \mathfrak{X} .
$$

For each $e \in \Sigma$

$$
x^{*} E(e) x_{i}=\lim _{\alpha} x^{*} S\left(g_{i}^{\alpha} \cdot k_{e}\right) x_{i},
$$

and the Radon-Nikodym theorem yields functions $\left[f_{1}, \cdots, f_{n}\right]$ such that

$$
x^{*} E(e) x_{i}=\int_{e} f_{i}(\omega) \nu_{i}(d \omega), \quad e \in \Sigma,
$$

yielding (a). The argument proceeds much as before to yield (b) and the continuity of $U$ for the norm topologies.

Suppose now that $z_{\alpha}^{*}(x) \rightarrow z_{0}^{*}(x)$ for each $x \in \mathfrak{X}$. Let $\left[h_{1}, \cdots, h_{n}\right]$ be any $n$-tuple of bounded Borel functions. Then for each $i=1, \cdots, n$

(') The symbol $\bigvee \mathfrak{N}\left(x_{i}^{*}\right)$ denotes the least $\mathfrak{X}$-closed linear manifold containing the manifolds $\mathfrak{N}\left(x_{i}^{*}\right), i=1, \cdots, n$. 


$$
z_{\alpha}^{*} S\left(h_{i}\right) x_{i} \rightarrow z_{0}^{*} S\left(h_{i}\right) x_{i} .
$$

Let $U z_{\alpha}^{*}=\left[f_{1}^{\alpha}, \cdots, f_{n}^{\alpha}\right]$ and $U z_{0}^{*}=\left[f_{1}^{0}, \cdots, f_{n}^{0}\right]$. Then using Lemma 8.1, we have

$$
\begin{aligned}
z_{\alpha}^{*} S\left(h_{i}\right) x_{i} & =\lim _{m \rightarrow \infty} \sum_{j=1}^{n} S^{*}\left(f_{j}^{\alpha} \cdot k_{e_{m}}\right) x_{j}^{*} S\left(h_{i}\right) x_{i} \\
& =\lim _{m \rightarrow \infty} S^{*}\left(f_{i}^{\alpha} \cdot k_{e_{m}}\right) x_{i}^{*} S\left(h_{i}\right) x_{i} . \\
& =\lim _{m \rightarrow \infty} \int_{e_{m}} f_{i}^{\alpha}(\omega) h_{i}(\omega) \nu_{i}(d \omega) \\
& =\int_{\Omega} f_{i}^{\alpha}(\omega) h_{i}(\omega) \nu_{i}(d \omega) .
\end{aligned}
$$

Similarly

$$
z_{0}^{*} S\left(h_{i}\right) x_{i}=\int_{\Omega} f_{i}^{0}(\omega) h_{i}(\omega) \nu_{i}(d \omega)
$$

Consequently,

$$
\lim _{\alpha} \int_{\Omega} f_{i}^{\alpha}(\omega) h_{i}(\omega) \nu_{i}(d \omega)=\int_{\Omega} f_{i}^{0}(\omega) h_{i}(\omega) \nu_{i}(d \omega), \quad i=1, \cdots, n
$$

showing the continuity of $U$ when $\mathfrak{X}^{*}$ has its $\mathfrak{X}$-topology and $\sum_{i=1}^{n} L_{1}\left(\Omega, \Sigma, \nu_{i}\right)$ has its weak topology.

8.3. CoRollary. Under the hypotheses of Theorem 8.2, $\left[\mathrm{V}_{i \in \sigma} \mathfrak{R}\left(x_{i}^{*}\right)\right]$ $\cap\left[\mathrm{V}_{j \in \sigma^{\prime}} \mathfrak{N}\left(x_{j}^{*}\right)\right]=(0)$ for any partition $\left[\sigma, \sigma^{\prime}\right]$ of the set $[1, \cdots, n]$.

We are now able to prove the following important theorem.

8.4. Theorem. Let $\mathfrak{B}$ be a complete B.A. of projections in a Banach space $\mathfrak{X}$ and let $\mathfrak{B}^{*}$ be the $B$.A . of adjoints in $\mathfrak{B}^{*}$. Then a projection $E$ in $\mathfrak{B}$ has finite uniform multiplicity $n$ if and only if its adjoint $E^{*}$ in $\mathfrak{B}^{*}$ has finite uniform multiplicity $n$.

Proof. It is sufficient to suppose $E$ and $E^{*}$ satisfy the countable chain condition. Also since each projection is the union of projections of uniform multiplicity, it is enough to show (a) if $E$ has finite uniform multiplicity $n$, then $m\left(E^{*}\right) \leqq n$; and (b) if $E^{*}$ has finite uniform multiplicity $n$, then $m(E)$ $\leqq n$. To prove (a) suppose $E \mathfrak{X}=\mathrm{V}_{i=1}^{n} \mathfrak{M}\left(x_{i}\right)$ and choose $x_{i}^{*}$ as in Lemma 5.1. If $E^{*} \mathfrak{X}^{*} \neq \mathrm{V}_{i=1}^{n} \mathfrak{R}\left(x_{i}^{*}\right)$, then by the Hahn-Banach theorem for the $\mathfrak{X}$-topology of $\mathfrak{X}^{*}$ there exists a nonzero vector $x \in E \mathfrak{X}$ such that $y^{*} x=0$ for $y^{*} \in \mathrm{V}_{i=1}^{n} \mathfrak{N}\left(x_{i}^{*}\right)$. Then $x_{i}^{*} E(e) x=0, e \in \Sigma, i=1, \cdots, n$. Thus $x=0$ by the one-to-one character 
of the map $T$ of Theorem 5.2. The proof of (b) follows in the same way from the fact $U$ is one-to-one (Theorem 8.2).

8.5. Corollary. If $n$ is an integer and $E \in \mathfrak{B}$, then $m(E)=n$ if and only if $m\left(E^{*}\right)=n$.

8.6. Corollary. If $\mathfrak{X}$ is separable then $m(E)=m\left(E^{*}\right)$ for every $E \in \mathfrak{B}$.

Proof. For each integer $n$ let $E_{n}$ and $F_{n}^{*}$ be respectively the projections of uniform multiplicity $n$ of Theorems 3.4 and 6.4. Then $F_{n}^{*}=E_{n}^{*}$, the adjoint of $E_{n}$, in view of Theorem 8.4. Since $\mathfrak{X}$ is separable $E_{0}=I-\bigvee_{n=1}^{\infty} E_{n}$ is zero or has uniform multiplicity $\boldsymbol{\aleph}_{0}$. To complete the proof it is sufficient to prove that if $E_{0} \neq 0$, then $m\left(E_{0}^{*}\right)=\boldsymbol{\aleph}_{0}$. However, $m\left(E_{0}^{*}\right) \leqq \boldsymbol{\aleph}_{0}$ since if $\mathfrak{X}$ is separable, it is well known that the unit sphere in $\mathfrak{X}^{*}$ is compact metric and hence separable in the $\mathfrak{X}$-topology. By definition of the projections $F_{n}^{*}, E_{0}^{*}$ can have no part of finite nonzero multiplicity.

9. Similarity equivalence. We recall (cf. [7]) that a bounded linear operator $Q$ on a complex Banach space is a spectral operator of scalar type if it possesses a resolution of the identity $E(\cdot)$ defined on the Borel sets $\Sigma$ of the complex plane $\mathfrak{B}$ and $Q=\int_{\sigma(Q)} \lambda E(d \lambda)$, where $\sigma(Q)$ is the spectrum of $Q$. The resolution of the identity is a projection valued measure which is countably additive in the strong operator topology and satisfies

$$
\begin{array}{cc}
E(\mathfrak{B})=I, E(\phi)=0, E(e) Q=Q E(e), & e \in \Sigma \\
\sigma(Q ; E(e) \mathfrak{X}) \subseteq \bar{e}, & e \in \Sigma,
\end{array}
$$

where $\sigma(Q ; E(e) \mathfrak{X})$ denotes the spectrum of the restriction of $Q$ to the subspace $E(e) \mathfrak{X}$. If we denote the range of $E(\cdot)$ by $\mathfrak{B}$, then $\mathfrak{B}$ is a $\sigma$-complete B.A. of projections and its closure $\overline{\mathfrak{B}}^{s}$ in the strong operator topology is complete (cf. [3]). Thus the multiplicity theory of the preceding sections is applicable to $\overline{\mathfrak{B}}^{s}$. We now impose the assumption that $\overline{\mathfrak{B}}^{\text {s }}$ contains no projections of infinite uniform multiplicity. Our objective will be to show that, with this restriction, the operator $Q$ is, in a suitable sense, similarity equivalent to a normal operator on a Hilbert space. However, rather than to seek the maximum generality it will be convenient to suppose that $\mathfrak{B}$ is itself complete and satisfies the countable chain condition. Both these properties hold for $\mathfrak{B}$ if $\mathfrak{X}$ is separable, so this will be assumed for the rest of this section.

By Theorem 3.4 there exist disjoint projections $E_{n}, n=1,2, \cdots$, such that $I=\bigvee_{n=1}^{\infty} E_{n}$ and each $E_{n}$ is either zero or has uniform multiplicity $n$. (By our assumption above $E_{\boldsymbol{N}_{0}}=0$.) Corresponding to each $n$ there is a Borel set $e_{n}$ in the plane such that $E_{n}=E\left(e_{n}\right)$. We may suppose the sets $e_{n}$ are disjoint and $\mathfrak{B}=\cup_{n=1}^{\infty} e_{n}$.

Now consider $n$ fixed and suppose $E_{n} \neq 0$. It follows from Lemma 5.1 and Theorem 5.2 that there exist vectors $x_{1}, \cdots, x_{n}$ and functionals $x_{1}{ }^{*}, \cdots, x_{n}^{*}$ such that $E_{n} \mathfrak{X}=\bigvee_{i=1}^{n} \mathfrak{M}\left(x_{i}\right), \quad x_{i}^{*}\left(\bigvee_{j \neq i} \mathfrak{M}\left(x_{j}\right)\right)=0$, and the measures $\mu_{i}$ 
$=x_{i}^{*} E(\cdot) x_{i}$, now defined on the Borel sets of the plane, are positive and vanish outside $e_{n}$. Moreover, there is a natural continuous linear map $T_{n}$ of $E_{n} \mathfrak{X}$ into $\sum_{i=1}^{n} L_{1}\left(\mathfrak{B}, \Sigma, \mu_{i}\right)$ with densely lefined inverse. Let $W_{n}$ denote the identity map of $\mathfrak{W}_{n}=\sum_{i=1}^{n} L_{2}\left(\mathfrak{B}, \Sigma, \mu_{i}\right)$ into $\sum_{i=1}^{n} L_{1}\left(\mathfrak{B}, \Sigma, \mu_{i}\right)$. As the measures $\mu_{i}$ are finite, $W_{n}$ is defined and continuous, and the map $A_{n}=W_{n}^{-1} T_{n}$ is a densely defined closed map of $E_{n} \mathfrak{X}$ into $\mathfrak{W}_{n}$ with densely defined inverse. We suppose the norm of $\left[h_{1}, \cdots, h_{n}\right]$ in $\mathfrak{S}_{n}$ is defined to be

$$
\left[\sum_{i=1}^{n} \int_{\mathfrak{B}}\left|h_{i}(\lambda)\right|^{2} \mu_{i}(d \lambda)\right]^{1 / 2}
$$

so that $\mathfrak{W}_{n}$ is a Hilbert space. It is easily seen that

$$
\begin{aligned}
D\left(A_{n}\right) & =\left\{x \in E_{n} \mathfrak{X} \mid\left(T_{n} x\right)_{i}(\cdot) \in L_{2}\left(\mathfrak{B}, \Sigma, \mu_{i}\right), i=1, \cdots, n\right\}, \\
D\left(A_{n}^{-1}\right) & =\left\{\left[h_{1}, \cdots, h_{n}\right] \in \mathfrak{W}_{n} \mid \lim _{m \rightarrow \infty} \sum_{i=1}^{n} S\left(h_{i} \cdot k_{\epsilon_{m}}\right) x_{i} \text { exists }\right\},
\end{aligned}
$$

where $e_{m}=\left\{\lambda|| h_{i}(\lambda) \mid \leqq m, i=1, \cdots, n\right\}$. Moreover, if $g$ is a bounded Borel function on $\mathfrak{B}$, the closure in $\mathfrak{S}_{n}$ of the densely defined operator $A_{n} S(g) A_{n}^{-1}$ sends $\left[h_{1}, \cdots, h_{n}\right]$ into $\left[g h_{1}, \cdots, g h_{n}\right]$, i.e. corresponds to multiplication by $g$. Taking for $g$ the characteristic function $k_{e}$ of a Borel set there results an isomorphic correspondence $E(e) \rightarrow k_{e}$ between subprojections of $E_{n}$ in $\mathscr{B}$ and a B.A. $\tilde{\mathfrak{B}}_{n}$ of self adjoint projections in $\mathfrak{S}_{n}$. Moreover, $\tilde{\mathfrak{B}}_{n}$ is the resolution of the identity of the bounded normal operator $\widetilde{Q}_{n}=\int_{e_{n}} \lambda \tilde{E}(d \lambda)$ which is the closure of $A_{n} E\left(e_{n}\right) Q A_{n}^{-1}$.

Now let the Hilbert space $\mathfrak{S}=\sum_{n=1}^{\infty} \mathfrak{W}_{n}$ be the direct sum of the Hilbert spaces $\mathfrak{W}_{n}$. Elements of $\mathfrak{S}$ will be denoted by the single letter $h$. We shall define a map $A$ of $\mathfrak{X}$ into $\mathfrak{S}$. Let

$$
D(A)=\left\{x \mid E_{n} x \in D\left(A_{n}\right) \text { for all } n \text { and } \sum_{n=1}^{\infty} A_{n} E_{n} \cdot x \text { converges in } \mathfrak{S}\right\} .
$$

Define

$$
A x=\sum_{n=1}^{\infty} A_{n} E_{n} x, \quad x \in D(A) .
$$

The properties of $A$ are collected in the next lemma.

9.1. Lemma. The operator $A$ is a densely defined closed linear map of $\mathfrak{X}$ into $\mathfrak{W}$ with densely defined inverse. Moreover, if $P_{n}$ denotes the perpendicular projection of $\mathfrak{S}_{\mathrm{S}}$ onto $\mathfrak{S}_{n}$, then

$$
D\left(A^{-1}\right)=\left\{h \mid P_{n} h \in D\left(A_{n}^{-1}\right) \text { for all } n \text { and } \sum_{n=1}^{\infty} A_{n}^{-1} P_{n} h \text { converges in } \mathfrak{X}\right\}
$$




$$
A^{-1} h=\sum_{n=1}^{\infty} A_{n}^{-1} P_{n} h, \quad h \in D\left(A^{-1}\right) .
$$

Proof. Let $y_{m} \in D(A), y_{m} \rightarrow y_{0}$ and $A y_{m} \rightarrow h_{0}$. Then $E_{n} y_{m} \rightarrow E_{n} y_{0}$, and $P_{n} A y_{m}=A_{n} E_{n} y_{m} \rightarrow P_{n} h_{0}, n=1,2, \cdots$. Since $A_{n}$ is closed $E_{n} y_{0} \in D\left(A_{n}\right)$ and $A_{n} E_{n} y_{0}=P_{n} h_{0}$. Now

$$
\sum_{n=1}^{\infty} A_{n} E_{n} y_{0}=\sum_{n=1}^{\infty} P_{n} h_{0}=h_{0} .
$$

Thus $y_{0} \in D(A)$ and $A y_{0}=h_{0}$, showing $A$ is closed. If $A x=0$ then $A_{n} E_{n} x$ $=E_{n} A x=0$. Since $A_{n}^{-1}$ exists $E_{n} x=0$, so $x=\sum_{n=1}^{\infty} E_{n} x=0$ showing $A^{-1}$ exists. Since each $A_{n}^{-1}$ has dense domain the same is true of $A^{-1}$. The formula for $D\left(A^{-1}\right)$ is easily verified.

The next theorem is a straightforward consequence of the preceding discussion.

9.2. Theorem. Let $\mathfrak{X}$ be a separable complex Banach space and $Q$ be a bounded scalar type spectral operator in $\mathfrak{X}$. Let $\mathfrak{B}$ denote the range of the resolution of the identity $E(\cdot)$ of $Q$ and suppose that $B$ contains no projection of infinite uniform multiplicity. If $A$ is the closed densely defined linear map of $\mathfrak{X}$ into the Hilbert space $\mathfrak{S}$ of Lemma 9.1, then the closure $\widetilde{Q}$ of $A Q A^{-1}$ is a bounded normal operator. For each bounded Borel function $g$ on the plane let $\widetilde{S}(g)$ denote the closure of $A S(g) A^{-1}$. The correspondence $\tau: S(g) \rightarrow \widetilde{S}(g)$ preserves the operational calculus and

$$
\widetilde{Q}=\int_{\sigma(Q)} \lambda \tilde{E}(d \lambda)
$$

where $\tau E(e)=\tilde{E}(e), e \in \Sigma$.

Our final objective is to show that the operator $A$ induces a one to one correspondence betwen the subspaces of $\mathfrak{X}$ invariant under $\mathfrak{B}$ and those of $\mathfrak{S}$ invariant under $\tilde{\mathfrak{B}}$, the resolution of the identity of $Q$.

9.3. Definition. If $\mathfrak{M}$ is a closed invariant subspace in $\mathfrak{X}$ we denote by $\Phi(\mathfrak{M})$ the closure in $\mathfrak{S}$ of the linear set $A(\mathfrak{M} \cap D(A))$. Similarly if $\Omega$ is a closed invariant subspace in $\mathfrak{E}, \Psi(\Re)$ denotes the closure in $\mathfrak{X}$ of $A^{-1}\left(\Re \cap D\left(A^{-1}\right)\right)$.

It follows from Theorem 5.2(b) and Lemma 9.1 that $\mathfrak{M} \cap D(A)$ is dense in $\mathfrak{M}$. Similarly $\mathfrak{R} \cap D\left(A^{-1}\right)$ is dense in $\Re$. It is conceivable that $\Phi(\mathfrak{M})$ contains elements of $D\left(A^{-1}\right)$ not belonging to $A(\mathfrak{M} \cap D(A))$, in which case $\Psi(\Phi(\mathfrak{M})$ ) contains $\mathfrak{M}$ properly. Our objective is to show that this does not happen. In fact $\Psi(\Phi(\mathfrak{M}))=\mathfrak{M}, \Phi(\Psi(\Re))=\Re$, for all $\mathfrak{M}$ and $\Omega$. This property of the invariant subspaces is a consequence of the special structure of $E_{n} \mathfrak{X}$, $n=1,2, \cdots$, and it will be convenient to prove a lemma first about the maps $A_{n}$. 
9.4. Lemma. (a) If $\mathfrak{M}$ is an invariant subspace of $E_{n} \mathfrak{X}$ then

$$
\Phi(\mathfrak{M}) \cap D\left(A_{n}^{-1}\right) \subseteq A_{n}\left[\mathfrak{M} \cap D\left(A_{n}\right)\right] .
$$

(b) If $\Omega$ is an invariant subspace of $\mathfrak{E}_{n}$ then

$$
\Psi(\Re) \cap D\left(A_{n}\right) \subseteq A_{n}^{-1}\left[\Re \cap D\left(A_{n}^{-1}\right)\right] .
$$

Proof. To prove (a) suppose $h^{0}=\left[h_{1}^{0}, \cdots, h_{n}^{0}\right]$ is an element of $\Phi(\mathfrak{M})$ $\cap D\left(A_{n}^{-1}\right) \subseteq \mathfrak{S}_{n}$, and that $h^{0}=A_{n} y_{0}$. We must show $y_{0} \in \mathfrak{M}$. There exist vectors $y_{m}$ in $\mathfrak{M} \cap D\left(A_{n}\right)$ such that $A_{n} y_{m}=\left[h_{1}^{m}, \cdots, h_{n}^{m}\right] \rightarrow\left[h_{1}^{0}, \cdots, h_{n}^{0}\right]$ in $\mathfrak{S}_{n}$ $=\sum_{i=1}^{n} L_{2}\left(\mathfrak{B}, \Sigma, \mu_{i}\right)$. By extracting subsequences we may suppose $h_{i}^{m} \rightarrow h_{i}^{0}$ almost uniformly with respect to $\mu_{i}, i=1, \cdots, n$. Given $\epsilon>0$ there exists a Borel set $e_{\epsilon}$ such that $\mu_{i}\left(e_{\epsilon}^{\prime}\right)<\epsilon, i=1, \cdots, n$, and $h_{i}^{m} \rightarrow h_{i}^{0}$ uniformly on $e_{\epsilon}$. The functions $h_{i}^{m}$ are bounded on $e_{e}$ and

$$
\begin{aligned}
E\left(e_{\epsilon}\right) y_{m} & =\sum_{i=1}^{n} S\left(h_{i}^{m} \cdot k_{e_{\epsilon}}\right) x_{i} \\
& \rightarrow \sum_{i=1}^{n} S\left(h_{i}^{0} \cdot k_{e_{\epsilon}}\right)=E\left(e_{\epsilon}\right) y_{0},
\end{aligned}
$$

so $E\left(e_{\epsilon}\right) y_{0} \in \mathfrak{M}$. An obvious limiting argument shows $y_{0} \in \mathfrak{M}$, establishing (a).

If $y_{0} \in \Psi(\Re) \cap D\left(A_{n}\right)$ and $y_{0}=A_{n}^{-1} h_{0}$, there is a sequence $\left\{h^{m}\right\}$ of $n$-tuples in $\Omega \cap D\left(A^{-1}\right)$ such that $y_{m}=A_{n}^{-1} h^{m} \rightarrow y_{0}$. Then $T_{n} y_{m}=\left[h_{1}^{m}, \cdots, h_{n}^{m}\right]$ $\rightarrow\left[h_{1}^{0}, \cdots, h_{n}^{0}\right]$ in $\sum_{i=1}^{n} L_{1}\left(\mathfrak{B}, \Sigma, \mu_{i}\right)$. Consequently, given $\epsilon>0, \tilde{E}\left(e_{\epsilon}\right) h^{m}$ $\rightarrow \tilde{E}\left(e_{\epsilon}\right) h^{0}$ in subsequence, where $\mu_{i}\left(e_{\epsilon}^{\prime}\right)<\epsilon, i=1, \cdots, n$. Thus $h_{0} \in \Re$, so $y_{0} \in A_{n}^{-1}\left[\AA \cap D\left(A_{n}^{-1}\right)\right]$.

9.5. THEOREM. Let the hypotheses of Theorem 9.2 be satisfied and let the maps $\Phi$ and $\Psi$ be defined as in Definition 9.3. Then there exists a one to one correspondence between the invariant subspaces $\mathfrak{M}$ of $\mathfrak{X}$ and the invariant subspaces $\Re$ of $\mathfrak{S}$ given by the relations

$$
\Psi(\Phi(\mathfrak{M}))=\mathfrak{M}, \quad \Phi(\Psi(\Re))=\Re, \quad \mathfrak{M} \subseteq \mathfrak{X}, \quad \Re \subseteq \mathfrak{G} .
$$

Proof. The desired relations follow immediately from the two equations

$$
\Phi(\mathfrak{M}) \cap D\left(A^{-1}\right)=A(\mathfrak{M} \cap D(A))
$$

and

$$
\Psi(\Re) \cap D(A)=A^{-1}\left(\Re \cap D\left(A^{-1}\right)\right) .
$$

We shall prove (a), the proof of (b) being similar. Clearly the right side of (a) is contained in the left. It follows from Lemma 9.4 that

$$
\Phi\left(E_{n} \mathfrak{M}\right) \cap D\left(A^{-1}\right) \subseteq A\left[\left(E_{n} \mathfrak{M}\right) \cap D(A)\right], \quad n=1,2, \cdots .
$$

Now $\left(E_{n} \mathfrak{M}\right) \cap D(A)=E_{n}(\mathfrak{M} \cap D(A))$ and $P_{n} A(\mathfrak{M} \cap D(A))=A E_{n}(\mathfrak{M} \cap D(A))$. 
Taking closures of both sides of the last equation shows $P_{n} \Phi(\mathfrak{M})=\Phi\left(E_{n} \mathfrak{M}\right)$, $n=1,2, \cdots$. Now let $h \in \Phi(\mathfrak{M}) \cap D\left(A^{-1}\right)$ and $h=A x$. For each $n$

$$
P_{n} h \in P_{n} \Phi(\mathfrak{M})=\Phi\left(E_{n} \mathfrak{M}\right) \text {. }
$$

Thus by (\#)

$$
A^{-1} P_{n} h=E_{n} x \in E_{n} \mathfrak{M},
$$

so

$$
x=\sum_{n=1}^{\infty} E_{n} x \in \mathfrak{M} \cap D\left(A^{-1}\right) .
$$

10. Unsolved problems. The main question left open is the relation between the multiplicity functions in $\mathscr{B}$ and $\mathfrak{B}^{*}$ on projections of infinite multiplicity. It was shown in Corollary 8.5 that $m(E)=m\left(E^{*}\right)$ for all projections of finite multiplicity. We have no information on this question. It is undecided even whether $E^{*}$ has infinite uniform multiplicity when $E$ has infinite uniform multiplicity. A related question concerns invariant subspaces. If $\mathfrak{M}$ is an invariant subspace we may define the multiplicity of $\mathfrak{B}$ in $\mathfrak{M}$. If $\mathfrak{M}_{1} \subseteq \mathfrak{M}_{2}$ it is to be expected that $m_{1}(E) \leqq m_{2}(E), E \in \mathfrak{B}$. This is true if $\mathfrak{B}$ contains no projections of infinite uniform multiplicity, but the question is open in general.

\section{BiBLIOGRAPHY}

1. W. G. Bade, Unbounded spectral operators, Pacific J. Math. vol. 4 (1954) pp. 373-392. 393-414.

2. - Weak and strong limits of spectral operators, Pacific J. Math. vol. 4 (1954) pp.

3. - On Boolean algebras of projections and algebras of operators, Trans. Amer. Math. Soc. vol. 80 (1955) pp. 345-360. 187.

4. J. Dieudonne, Sur la theorie spectrale, J. Math. Pures Appl. vol. 35 (9) (1956) pp. 175-

5. - Champs de vecteurs non localement triviaux, Arch. Math. vol. 7 (1956) pp. 6-10.

6. J. Dixmier, Le moyennes invariantes dans les semi-groupes et leurs applications, Acta Univ. Szeged vol. 12(A) (1950) pp. 213-227.

7. N. Dunford, Spectral operators, Pacific J. Math. vol. 4 (1954) pp. 321-354.

8. N. Dunford and J. Schwartz, Linear operators, II. Spectral theory, Interscience, forthcoming.

9. P. R. Halmos, Introduction to Hilbert space and the theory of spectral multiplicity, New York, 1951.

10. J. L. Kelley, Commutative operator algebras, Proc. Nat. Acad. Sci. U.S.A. vol. 38 (1952) pp. $598-605$.

11. G. W. Mackey, Commutative Banach algebras, multigraphed Harvard lecture notes, ed. by A. Blair, 1952.

\section{UNiversity of California, Berkeley, California}

